\title{
Esthetic Rehabilitation of Bilateral Geminated Teeth: A Case Report
}

\author{
Süha Türkaslan ${ }^{a}$ \\ Hasan Suat Gökçe \\ Mehmet Dalkız
}

\begin{abstract}
Geminated teeth are the consequences of developmental anomalies leading to the eruption of joined elements. According to current definitions, gemination occurs when one tooth bud tries to divide, while fusion occurs if two buds unite. Clinical experience shows, however that diagnosis can be complicated due to superimposed anomalies. This report describes a unique case of bilateral gemination of permanent maxillary central incisors. The esthetic rehabilitation of the geminated incisors accomplished utilizing all ceramic crowns. It is important that in these types of cases, reaching to the available esthetics and avoiding the complication of caries and periodontal problems with prosthetic application is satisfactory. (Eur J Dent 2007;1:188-191)
\end{abstract}

Key Words: Gemination; Double teeth; Fusion; Permanent teeth; Developmental anomalies; Maxillary central incisors.

\section{INTRODUCTION}

Developmental dental disorders may be due to abnormalities in the differentiation of the dental lamina and the tooth germs lanomalies in number, size and shape) or to abnormalities in the formation of the dental hard tissues lanomalies in structure). In some, both stages of differen-

n a Assistant Professor, Suleyman Demirel University, Faculty of Dentistry,

Department of Prosthodontics, Isparta - Turkey

b Assistant Professor, Gulhane Military Medical Academy, Dental Sciences Center,

Department of Prosthodontics, Ankara - Turkey

Associate Professor, Gulhane Military Medical

Academy, Dental Sciences Center,

Department of Prosthodontics, Ankara - Turkey

- Corresponding Author:

Assist. Prof. Dr. Süha TÜRKASLAN

Süleyman Demirel University, Faculty of Dentistry

Department of Prosthodontics Isparta / TURKEY

e-mail: suhaturkaslandyahoo.com

Tel : 009002462113263 Fax : 009002462370607 tiation are abnormal. Developmental dental disorders are not only congenital but they may also be inherited, acquired or idiopathic. The terms "double tooth", "double formations", joined teeth", or "fused teeth" are often used to describe gemination and fusion, both of which are primary developmental abnormalities of the teeth. ${ }^{1-7}$ In the primary dentition, the frequency of gemination or fusion is about $2.5 \%$. Bilateral presentation is very rare. A survey of the literature has revealed prevalence estimates for bilateral double teeth ranging from 0.01 to $0.04 \%$ in the primary, and $0.05 \%$ in the permanent dentition. ${ }^{1,7}$

Gemination is defined as an attempt to make two teeth from one enamel organ. This results in a structure with two completely or incompletely separated crowns with a single root and root canal. Occasionally we see complete cleavage or twinning (two teeth from one enamel organ). The etiology is unknown, but trauma has been suggested as a possible cause, though a familial tendency has been suggested. ${ }^{8}$ Gemination is observed in the deciduous as well as in the permanent denti- 
tion. 3,4,7

In the anterior region, this anomaly can cause unpleasant esthetic appearance due to irregular morphology. If a deep groove is present, these teeth may be susceptible to caries and periodontal disease and may require endodontic intervention in some cases which may be complicated..$^{1,2}$

Gemination usually present asymptomatically. In fact, the co-operation of practitioners with expertise in multiple areas of dentistry is important to create or achieve functional and esthetic success in these cases. Several treatment methods have been described in the literature with respect to the different types and morphological variations of fused teeth, including endodontic, restorative, surgical, periodontal and/or orthodontic treatment. ${ }^{1,2,4,9-11}$

In clinical situations, cases of fusion have the appearance of a congenitally missing tooth, while in gemination the number of teeth in the dentition is normal, provided the double tooth is counted as one unit. ${ }^{1,4}$

This paper reports aesthetic rehabilitation of a rare case; bilateral gemination of permanent maxillary central incisors utilizing heat-pressed lithium disilicate all ceramic crowns.

\section{CASE REPORT}

A 22-year-old white male referred to our department. The patient complained about the large, unsightly maxillary central incisors, lip irritation and incising problems. The patient did not complain of previous painful symptoms in that region and his medical and dental histories were unremarkable. No other member of the family was affected with similar dental anomalies. Clinical examination revealed the presence of an irregular bilateral morphology of the permanent maxillary central incisors. The maxillary dental arch pre- sented two large central incisors that were abnormally wide and bifid crowns.

The incisors showed a buccal and palatal groove from the incisal edge to the cervical portion of the tooth. An initial caries lesion was noted on the labial groove of the right incisor, along with gross plaque accumulation (Figure 1). The periapical radiography showed each incisor had one root (Figure 2).

The left central incisor was caries-free, as were all other erupted teeth had no restoration in the panoramic radiographic examination but only first molars had proximal caries which are restored before the prosthetic treatment procedure (Figure 3). Left and right maxillary central incisors were found to be free from periodontal disease. The incisors responded to electrical pulp testing within normal limits.

Treatment plans for the patient included all ceramic crowns for the upper incisors and canines. After administering local anesthesia, preparations were made in the incisal, buccal, mesial, distal and palatinal surface of each of the clinical crowns in left and right maxillary central incisors, laterals and canines (Figure 4). The preparations were completed with full chamfer preparation. Anatomic preparations were conducted, especially in the distal portions of the maxillary central incisors wider preparations carried out in order to eliminate the amorphous shape of the maxillary central incisors. Nevertheless endodontic treatment of these teeth was not required.

The impressions of the prepared teeth were obtained utilizing polyether based elastomeric impression material (Impregum Penta H, Duosoft Grant L, Duosoft, 3M Espe, Germanyl After casting the model, all ceramic cores manufactured utilizing heat-press ceramic technique (IPS Empress 2, Ivoclar, Schaan, Liechtenstein) with \# 100 lithium
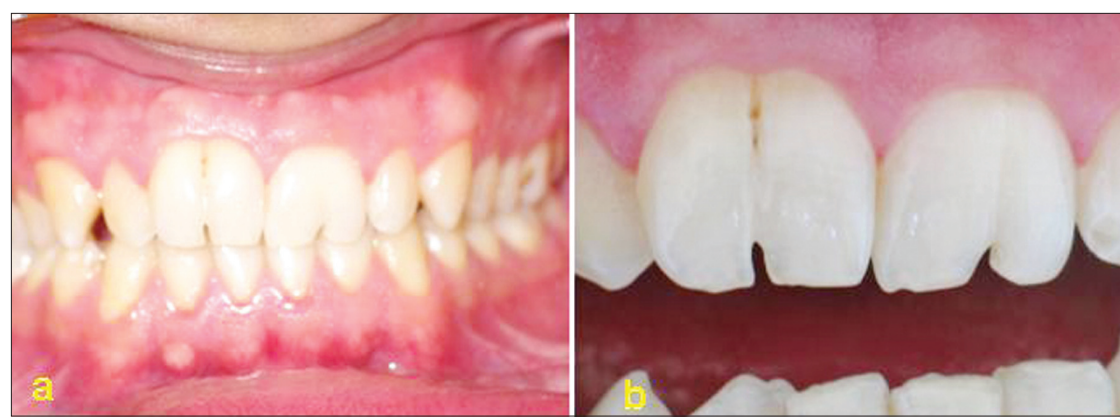

Figure 1. 1.The intraoral photographs of the case

a. Frontal view of the geminated teeth

b. Developmental grooves extending from incisal edge to the cemento-enamel junction

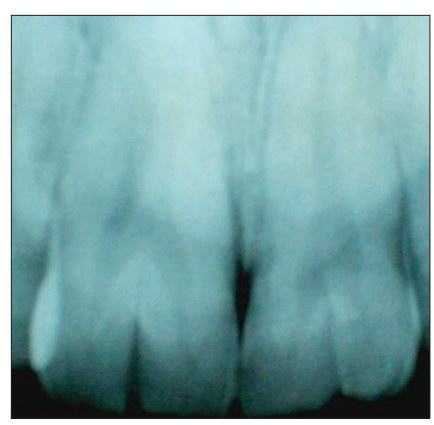

Figure 2. Periapical radiograph of the geminated incisors. 
disilicate ceramic ingots. After the heat-press procedure completed ceramic cores were ready for try in procedures (Figure 5). Final restoration conducted by layering technique (Empress 2 Dentine Ivoclar, Schaan, Liechtenstein). All ceramic crowns were bonded to the prepared abutments using dual cure composite resin luting cement (Rely-X ARC, 3M-ESPE, Germany). These fixed restoration treatment resulted in marked improvement in the esthetics of the anterior region and also enhanced periodontal health (Figure 6).

The patient was encouraged to practice strict oral hygiene. After a 3-month period, the patient returned for evaluation. At the 1-year recall appointment, the abutment teeth were symptomfree and periodontal tissues were healthy.

\section{DISCUSSION}

The terminology dental fusion and gemination are used to define two different morphological dental anomalies, characterized by the formation of a clinically wide tooth. Despite the considerable number of cases reported in the literature, the differential diagnosis between these abnormalities is difficult. Case history and clinical and radiographic examinations can provide the information required for the diagnosis of such abnormalities., ${ }^{1,3-6,12}$ After

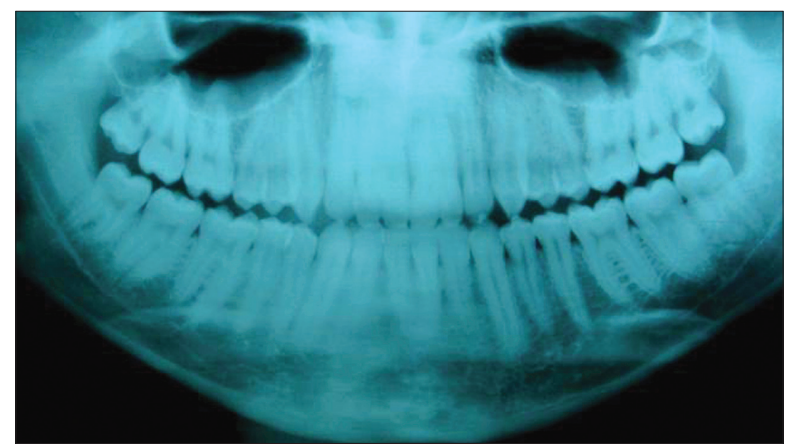

Figure 3. Panoramic radiographic image la full set of permanent teeth is present).

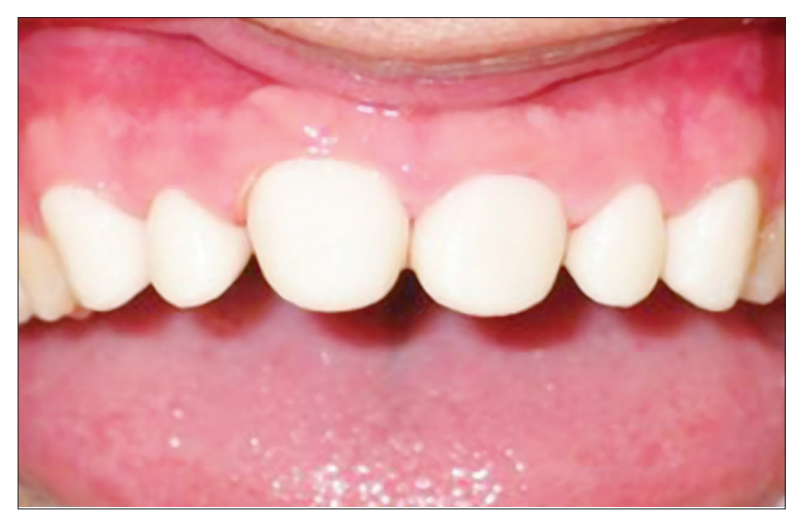

Figure 5. All ceramic cores during try-in procedure. a judicious evaluation of all information we can report that this case represents bilateral gemination of maxillary incisors.

While the literature on the occurrence of double teeth is extensive, there is still much discussion concerning the nomenclature. Some authors have tried to differentiate them by counting the teeth or by observing the root morphology: others use fusion and gemination as synonyms. Finally, some authors simply call the phenomenon "double teeth" or "connoted teeth" to avoid confusion over terminology. ${ }^{9,10}$

The use of Levitas' classification to distinguish between cases of fusion and gemination is very practical. ${ }^{9}$ The differential diagnosis between fusion and gemination, based on the number of teeth present on the dental arch, is not, however, always possible. ${ }^{5}$ This is because nothing impairs the fusion between a "normal" and a supernumerary element while the contiguous "normal" tooth is congenitally absent, resembling clinical cases of gemination. ${ }^{5,6}$

The phenomenon of gemination arises when two teeth develop from one tooth bud and, as a result, the patient has a larger tooth but a normal number, in contrast to fusion where the patient would appear to be missing one tooth. ${ }^{3,4,6,7}$ Fused

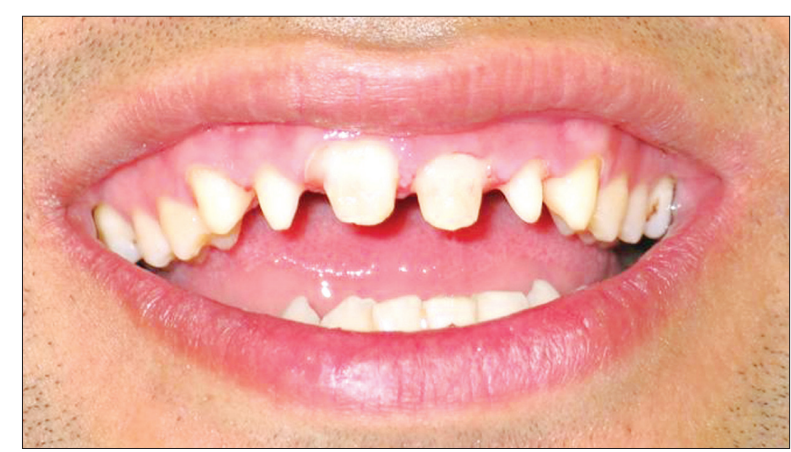

Figure 4. Frontal view of the prepared maxillary incisors and canines.

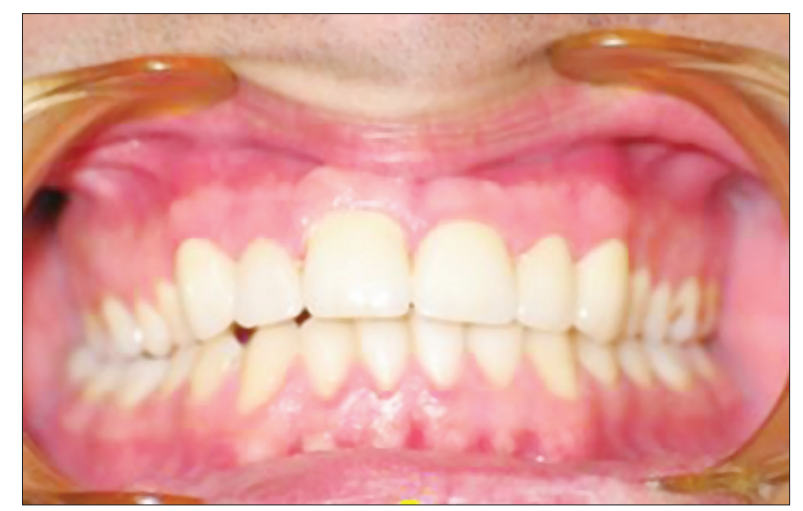

Figure 6. Frontal view of the cemented all ceramic crowns. 
teeth arise through union of two normally separated tooth germs, and depending upon the stage of development of the teeth at the time of union, it may be either complete or incomplete. On some occasions, two independent pulp chambers and root canals can be seen. However, fusion can also be the union of a normal tooth bud to a supernumerary tooth germ. In these cases, the number of teeth is also normal and differentiation from gemination may be very difficult, if not impossible. In geminated teeth, division is usually incomplete and results in a large tooth crown that has a single root and a single canal. ${ }^{1,3,5}$

This anatomic irregularity occurs more often in the deciduous than in the permanent dentition. Only a few cases of fusion involving molar and premolar teeth have been reported whereas, in both dentitions, the prevalence is higher in the anterior region. . $4,6,8,9$ Cases of bilateral fusion are less frequent than unilateral fusion. The anomaly can cause unpleasant esthetic appearance due to irregular morphology. When deep grooves are present, these teeth may be susceptible to caries and periodontal disease and may require endodontic intervention in some cases which may be complicated. 3,4,12,13

The main periodontal complication in gemination cases occurs due to the presence of fissures or grooves in the union between the teeth involved. If these defects are very deep and extend subgingivally, the possibility of bacterial plaque accumulation in this area is quite high. Strict oral hygiene is imperative to maintain periodontal health. Furthermore, gemination may have an adverse effect on occlusion, causing deviation and, sometimes, delaying the eruption of other teeth with aesthetic problems resulting from tooth. ${ }^{2}$ In this case, despite of the buccal grooves in the maxillary centrals no serious periodontal disease was evident.

The double teeth in the cases studied were mostly unilateral, involving two adjacent teeth. No difference was found in the proportion of double teeth in either the maxilla or mandible. ${ }^{1,2,6}$ In this case bilateral gemination was seen in maxilla which caused esthetic problems.

Difficult cases include a wide spectrum of problems. ${ }^{9-12}$ The best way to manage these difficult cases depends on a number of factors including the knowledge and technical skills of the practitioner. . $^{19-13}$

\section{CONCLUSIONS}

In this case, restoration of all the six maxillary anterior teeth was preferred due to the wide and amorphous shape of maxillary central incisors. For esthetic satisfaction, wideness of these teeth was divided and distributed and was shared among the lateral incisors and the canines.

Different cases require a variety of knowledge about alternative operative techniques and abilities. A multidisciplinary approach may contribute to the success of the treatment.

\section{REFERENCES}

1. Aguilo L, Gandia JL, Cibrian R, Catala M. Primary double teeth. A retrospective clinical study of their morphological characteristics and associated anomalies. Int J Paediatr Dent 1999;9:175-183.

2. Blank BS, Ogg RR, Levy AR. A fused central incisor. Periodontal considerations in comprehensive treatment. $J$ Periodontol 1985;56:21-24.

3. O Carroll MK. Fusion and gemination in alternate dentitions. Oral Surg 1990;69:655.

4. Maibaum WW. Fusion of confusion? Oral Surg Oral Med Oral Pathol 1990;69:656-657.

5. O'Reilly PMR. Structural and radiographic evaluation of four cases of tooth fusion. Aust Dent $J$ 1990;35:226-229.

6. Terezhalmy GT, Riley CK. Gemination/fusion. Quintessence Int 1999;30:437.

7. Duncan WK, Helpin ML. Bilateral fusion and gemination: a literature analysis and case report. Oral Surg Oral Med Oral Pathol. 1987;64:82-87.

8. Jarvinen S, Lehtinen L, Milen A. Epidemiologic study of joined primary teeth in Finnish children. Community Dent Oral Epidemiol. 1980;8:201-202.

9. Neves AA, Neves MLA, Farinhas JA. Bilateral connation of permanent mandibular incisors: a case report Int $J$ Paediatr Dent 2002;12:61-65.

10. Nunes E, de Moraes IG, de Novaes PMO, de Sousa SMG. Bilateral fusion of mandibular second molars with supernumerary teeth: Case Report. Braz Dent J 2002;13:137-141.

11. Velasco LF, de Araujo FB, Ferreira ES, Velasco LE. Esthetic and functional treatment of a fused permanent tooth: a case report. Quintessence Int 1997;28:677-680.

12. Milano M, Seybold SV, McCandless G, Cammarata R. Bilateral fusion of the mandibular primary incisors: report of case. ASDC J Dent Child 1999;66:280-282.

13. Peretz B, Brezniak N. Fusion of primary mandibular teeth. Report of case. ASDC J Dent Child 1992;59:366-368. 